\title{
Performance and Analysis of Voltage Scaled Repeaters for Multi-Walled Carbon Nanotubes as VLSI Interconnects
}

\author{
Jatinderpal \\ M.Tech Scholar \\ Department of Electronics and \\ Communication \\ Shaheed Bhagat Singh State \\ Technical Campus, Ferozepur, \\ India
}

\author{
Chakshu Goel \\ Assistant Professor \\ Department of Electronics and \\ Communication \\ Shaheed Bhagat Singh State \\ Technical Campus, Ferozepur, \\ India
}

\author{
Karamjit Singh Sandha \\ Assistant Professor \\ Department of Electronics and \\ Communication
}

Thapar University, Patiala, India

\begin{abstract}
Multi-walled carbon nanotubes (MWCNT) are promising candidates for futuristic Nano-electronic applications. MWCNT have potential to replace on-chip copper $(\mathrm{Cu})$ interconnects due to their large conductivity and current carrying capabilities. Delay is one of the major design constraints in very large scale integration (VLSI) circuits. This paper presents an analysis of propagation delay and effect of repeater insertion on propagation delay for both MWCNT and $\mathrm{Cu}$ interconnects at different technology nodes viz $32 \mathrm{~nm}$ and $22 \mathrm{~nm}$. In addition this paper deals with effect of voltage scaling in repeaters for long interconnects length in VLSI circuits in terms as propagation delay. It has been observed that propagation delay reduces with increase in bias voltage of the repeater at different interconnects length and technology nodes (32nm.22nm).
\end{abstract}

KEYWORDS- Carbon Nanotubes (CNT), Multi-walled CNT (MWCNT), Interconnects, Circuit Model.

\section{INTRODUCTION}

As VLSI technology advances over the years chip complexity increases and the feature size decreases [1]. Thus, both die size and device density of the VLSI circuits increase. The use of long interconnect lines (global interconnects) becomes essential due to the increased die size in VLSI chips. For these global interconnects, conventional interconnect technologies used by copper or aluminium fare badly because of their increasing resistively with length which causes some serious problems like electro migration and voids formation in the successive levels of interconnect paths [2]. For this reason, researchers introduced new materials such as carbon nanotubes (CNTs) which seem to be a possible solution for VLSI technologies of global interconnects.

CNTs are known as allotrope of carbon and made by rolling up a sheet of graphene into a cylinder. Structure of CNTs depends on chiral indices which are defined by direction of rolling a graphene sheet. CNTs can exhibit their unique armchair and zigzag structure which basically depends on the chiral indices $(n, m)$. For armchair CNTs, the chiral indices are defined by $n=m$ [4] and for zigzag CNTs, it is $n$ or $m=0$ [4]. For other values of $n$ and $m$, CNTs are known as chiral. Depending upon their different structures, CNTs can exhibit both metallic and semiconducting properties. By satisfying the condition $n-m=3 i$ (where $i$ is an integer), the armchair CNTs are always metallic and zigzag CNTs are either metallic or semiconducting in nature depending on their chiral indices [4] as shown in Fig. 1. Metallic CNTs have aroused a lot of interest as VLSI interconnects of the future [5] because of their extremely desirable electrical and thermal properties.

(a)

$(n, n)$

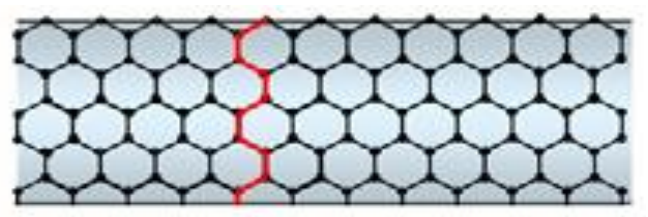

(b)

$(\mathrm{n}, 0)$

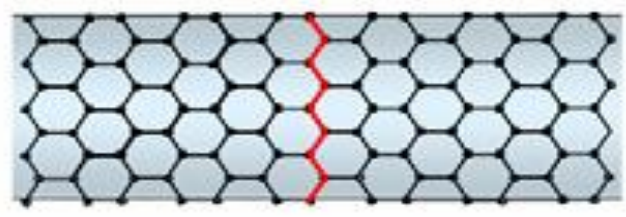

(c)

$(\mathrm{n}, \mathrm{m})$

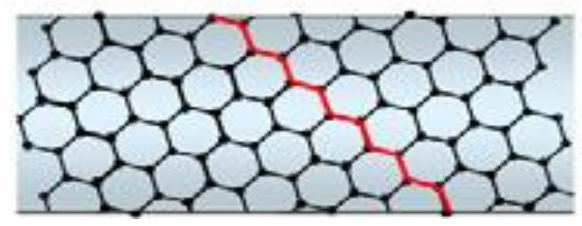

Fig.1. (a) Armchair metallic configuration. [6]. (b) Zigzag semiconducting configuration [6]. (c) Chiral Configuration

The two main types of CNTs[7] are Single-Walled Carbon Nanotubes (SWCNTs) and Multi-Walled Carbon Nanotubes (MWCNTs), as shown in Fig.2. A SWCNT is a single rolled layer of graphene, with a diameter between $0.4 \mathrm{~nm}$ and a few nanometres and a total length of several millimetres. If several 
SWCNTs with varying diameter are nested concentrically inside one another, the resulting structure is called a multiwalled carbon nanotube (MWCNT).[5]
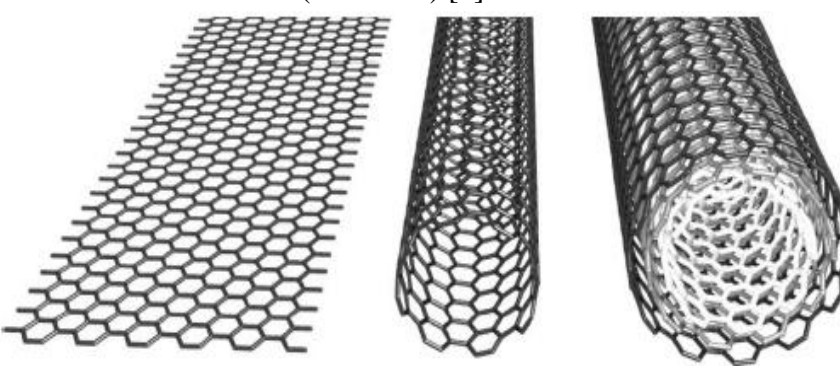

Fig. 2. Structure of a single layer of graphite (graphene) (left), single-walled

carbon nanotube (SWCNT) as a rolled-up graphene sheet (middle) and a multi-walled nanotube with three concentric shells (right).

This paper is organized as follows. The following Section 2 presents a discussion for a $\mathrm{Cu}$ interconnect and describes the models used for evaluating interconnect performance. Section 3 describes the equivalent circuit model for a MWCNT that is useful in the performance analysis of MWCNT interconnects and discusses existing work in the literature that compare MWCNT interconnects with copper. Section 4 analyses the impact of the repeaters on the performance of interconnects. Section 5 analyzes the impact of the voltage scaled repeater on the performance in terms of delay of interconnects. The results of the analysis of MWCNT and $\mathrm{Cu}$ interconnects are compared Section 6 and the lessons learned are summarized in Section 7.

\section{COPPER INTERCONNECTS}

A lot of work has been done in the field of copper interconnects regarding its circuit modeling and design methodologies. [5]

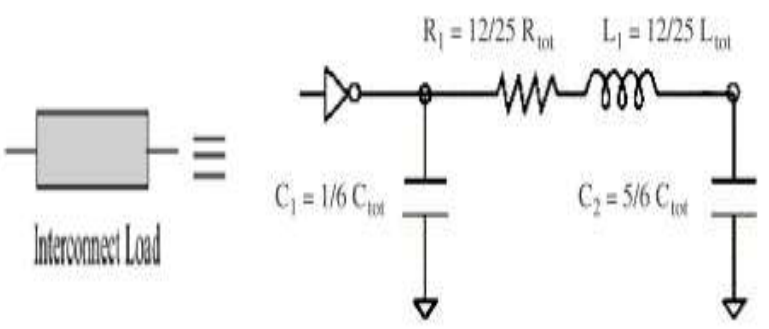

Fig.3 RLC II-model representation of an interconnect line

Performance analysis is done by circuit modeling interconnects into R, L and C parameters. $\Pi$-circuit has been used in this paper for modeling the Copper interconnects into $\mathrm{R}, \mathrm{L}$ and $\mathrm{C}$ parameters as shown in Fig.3 [5]. The Winbond TSM model Fig.4 [4], is for global layer interconnect lines with coupling above one ground. Here thickness of interconnect is t, width of CNT bundle is $\mathrm{w}$, height of the interconnect above the ground is h. Spacing between the interconnect $S$ is assumed to be equal to the interconnect width, i.e. $\mathrm{S}=\mathrm{W}$.

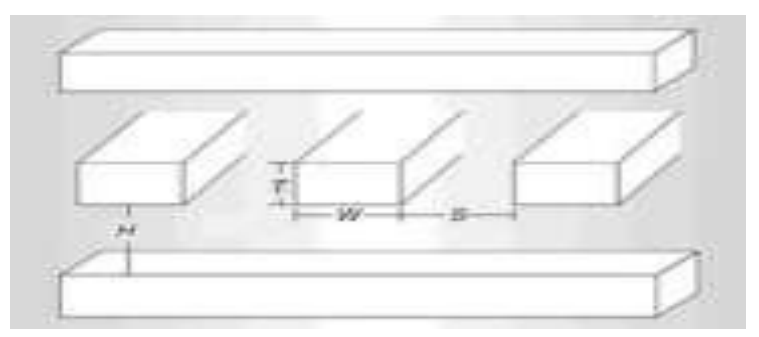

Fig.4 Geometry of Global Interconnects [5]

\section{MWCNT INTERCONNECTS}

An MWCNT consists of two or more SWCNTs with different diameters which are concentrically nested inside each other, as shown in Fig. 5, and may have diameters in a wide range of a few to hundreds of nanometers. The diameter of the outermost and innermost shells is $\mathrm{D}_{\max }$ and Dmin, respectively. The distance between center of nanotube and the ground plane is $H$. The spacing between shells corresponds to the van der Waals distance between graphene layers in graphite, which is $d \approx 0.34 \mathrm{~nm}$ [9].

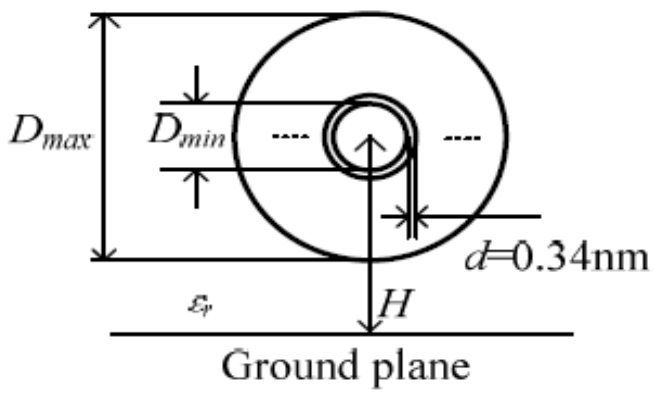

Fig.5. Cross section view of an MWCNT [10]

\subsection{Number of Channels}

The number of conducting channels for each shell [10] is

$$
N_{\text {chan } / \text { shell }}=\sum_{\text {subbands }} \frac{1}{\exp \left(\frac{\left|E_{i}-E_{F}\right|}{K_{B} T}\right)+1}
$$

where $E_{i}$, is the highest (or lowest) value for the sub-bands below (or above) the Fermi level $\mathrm{E}_{\mathrm{F}} . \mathrm{K}_{\mathrm{B}}$ and $\mathrm{T}$ are the Boltzmann constant and absolute temperature respectively. The number of channels per shell [11] can be approximated to

$$
N_{\frac{\text { chan }}{\text { shell }}}(D) \approx a . D+b, \text { for } D>3 \mathrm{~nm}
$$

where $D$ is the shell diameter, $a=0.0612 \mathrm{~nm}^{-1}$, and $b=0.425$. The number of shells are counted from outer to inner as 1 , $2, \ldots i, \ldots, M$. The ratio $\left(D_{\min } / D_{\max }\right)$ is equal to $1 / 2[10,11]$. Thus, the number of shells $n$ of the MWCNT is

$$
M=1+\operatorname{int}\left[\left(\frac{D_{\max }-D_{\max } / 2}{2 d}\right)\right]
$$

where "int [.]" indicates that only the integer part is taken into account. The diameter of the $\mathrm{i}^{\text {th }}$ shell is given by [10]

$$
D_{i}=D_{\text {max }}-2 d(i-1) \text {, for } 1 \leq i \geq M
$$

The innermost diameter in Fig. 5 is $D_{\min }=D_{\max }-2 d .(M-1)$.

Note that the ratio of $D_{\min } / D_{\max }$ is assumed to be $1 / 2, D_{\min }$ may be larger than $D_{\max } / 2$ because $D_{\max }$ may not be an integer multiple of $d$. The number of conducting channels of the $\mathrm{i}^{\text {th }}$ shell is given by [10]

$$
N_{i}=a \cdot D_{i}+b
$$


Hence, the total number of conducting channels is given by the sum of the conducting channels $(\mathrm{Ni})$ of all the shells. [10].

\subsection{Individual Shell Model}

Depending on different interconnect parasitic such as resistance, capacitance and inductance, $R L C$ circuit models as shown in Fig.6 for MWCNT is presented [12] on the basis of semi-classical one directional electron fluid model [13]. This 1-D electron fluid model is derived from a classical twodimensional electron fluid theory [13] taking into account electron-electron repulsive force.

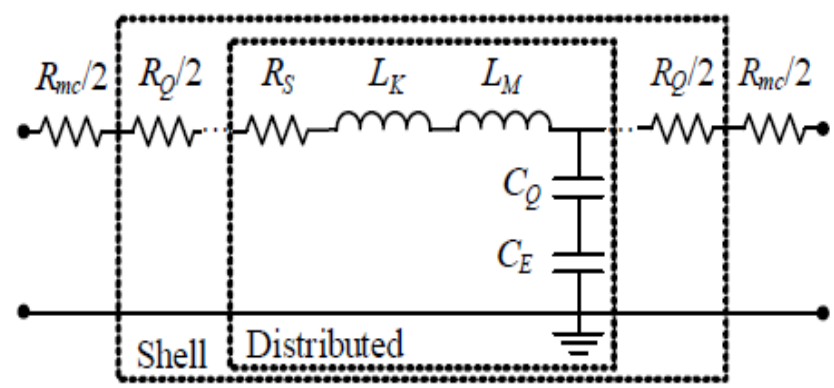

Fig.6. Equivalent circuit model of an individual shell [9]

\subsubsection{Resistance of shell- The total resistance of} a shell consists of three resistive elements[14]: scattering-induced resistance $R_{S}$ (considered only when the length of nanotube (shell) is larger than electron mean free path (MFP)), quantum contact resistance $\mathrm{R}_{\mathrm{Q}}$, and imperfect contact resistance $R_{m c}$. $R_{Q}$ and $R_{S}$ are intrinsic, and $R_{m c}$ is a function of the fabrication process. In the equivalent circuit model $\mathrm{R}_{\mathrm{Q}}$ and $\mathrm{R}_{\mathrm{mc}}$ are lumped elements, and $R_{S}$ is modelled as a distributed element. The effect of $R_{m c}$ is neglected in this paper because of its negligible small value. If shells of MWCNT have proper end contacts, these shells can contribute to conductance [15]. The total shell resistance [14] is given by

$$
R_{\text {shell }}=R_{Q}+R_{S} \cdot L=\frac{h}{2 e^{2} N}+\frac{h}{2 e^{2} N} \cdot \frac{L}{\lambda}
$$

Where $\mathrm{h} / 2 \mathrm{e}^{2}=12.9 \mathrm{k} \Omega$, and $L, \lambda$ and $N(=a . D+b)$ are the length, MFP and number of conducting channels of the shell respectively. It can be observed from (12) that the value of MFP plays an important role in determining the resistance of the nanotube. It has been proven that the MFP of metallic nanotube is directly proportional to the shell diameter (D). The MFP for metallic MWCNT at room temperature is

$$
\lambda=1000 . D
$$

3.2.2. Inductance: There are the two types of inductances for MWCNT i.e the magnetic and kinetic inductances per unit length [13] of a shell are given by

$$
\begin{gathered}
L_{\text {magnetic }}=\left(\frac{\mu}{2 \pi}\right) \cdot \cosh ^{-1}\left(\frac{2 H}{D}\right) \\
L_{K / \text { channel }}=h / 2 \times 2 v_{f} e^{2} \approx 8 n H / \mu m
\end{gathered}
$$

3.2.3. Capacitance: The capacitance of the MWCNT consists of two parts: quantum capacitance $C_{Q}$ and electrostatic capacitance $C_{E}$. The quantum capacitance per unit length of a shell is given by [13]

$$
\begin{aligned}
& C_{Q / \text { channel }}=2 \times 2 e^{2} / h v_{f} \approx 193 \mathrm{aF} / \mu \mathrm{m} \\
& C_{Q / \text { shell }}=C_{Q / \text { channel }} . N
\end{aligned}
$$

The electrostatic capacitance per unit length of a shell is given by

$$
C_{E}=\frac{2 \pi \varepsilon}{\cosh ^{-1}\left(\frac{2 H}{D_{\max }}\right)}
$$

The potentials of different shells cannot be assumed to be equal, which induces shell to shell capacitive coupling. The shell to shell capacitance per unit length (Cs) [10] can be obtained by using coaxial capacitance formula.

$$
C_{S}=\frac{2 \pi \varepsilon}{\ln \left(\frac{D_{\text {out }}}{D_{\text {in }}}\right)}=\frac{2 \pi \varepsilon}{\ln \left(D_{\text {out }} /\left(D_{\text {out }}-2 d\right)\right.}
$$

where Dout and Din are the outer and inner diameters of adjacent

coaxial shells respectively and $d=0.34 \mathrm{~nm}$.

\subsection{Equivalent Circuit Model of MWCNT}

Based on the above parameters, an equivalent distributed circuit model for MWCNT interconnect is shown in Fig. 6 [17]. The quantum capacitance $C_{Q}$ is in series with electrostatic capacitance (including shell-to-shell capacitance $C_{S}$ and ground capacitance $C_{E}$ ). Note that only one total ground capacitance $C_{E}$ is shown in Fig.7.

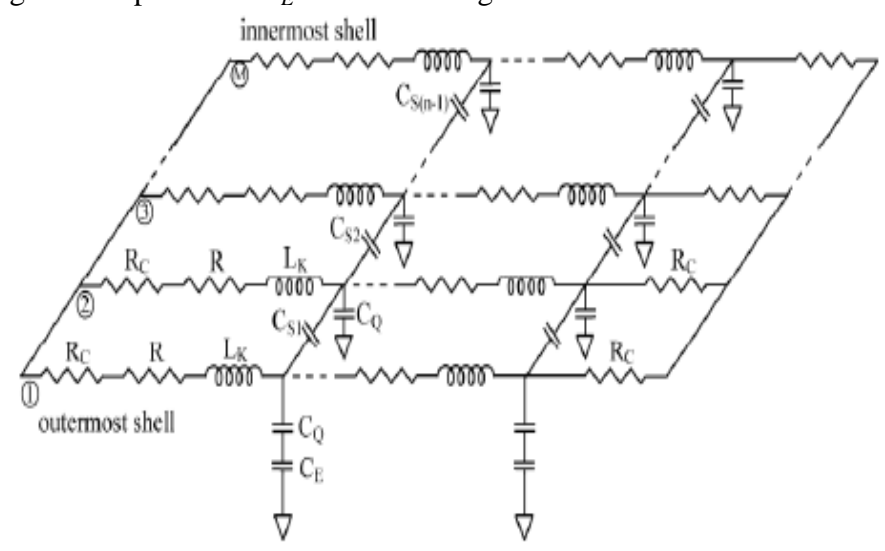

Fig.7. Equivalent circuit of a MWCNT interconnects [17].

\section{REPEATERS IN INTERCONNECTS}

The long interconnect lines give high propagation delays due to capacitive nodes thus degrading the performance of the device. To drive these high capacitive nodes buffers are needed. These buffers are also called repeaters. A basic interconnect circuit with one repeater is shown in Fig. 8 [6]. A repeater reduces the propagation delay by mitigating the charging-discharging effect of the capacitor [6].

But a single repeater offers a large RC (Resistive- Capacitive) load at the gate terminals connected to it. For driving long interconnects a number of repeaters have to be inserted at equal distances between the interconnect ends. Therefore, in long interconnects, the propagation delay reduces 
significantly by insertion of the repeaters at optimally spaced points along the line[14].

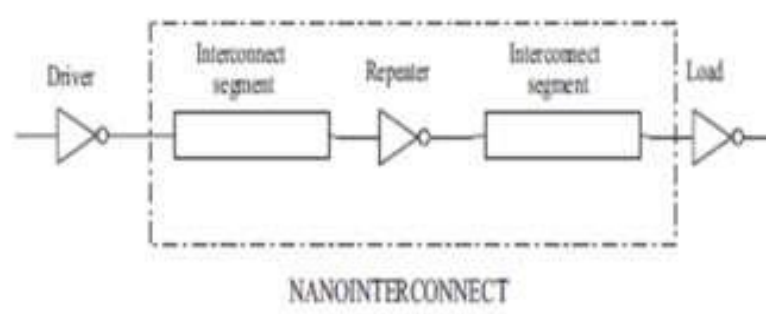

Fig.8 Basic Nano interconnect circuit with one repeater

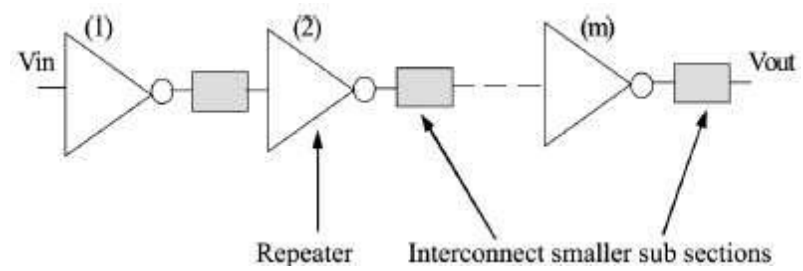

Fig.9 m number of repeaters driving an interconnect divided into subsections

Fig.9 [12] shows $m$ number of repeaters inserted in interconnect, divided into subsections. As interconnect is divided into subsections, the cumulative RC constant is reduced. However, the additional delay due to repeaters has to be taken into account. A lot of work has been done regarding CMOS inverters. These are the simplest buffers or repeaters in VLSI interconnect.

In this paper the effect of number of repeaters on the propagation delay for both MWCNT and Copper interconnects over the different technology nodes have been analyzed.

\section{VOLTAGE SCALED REPEATERS FOR GLOBAL INTERCONNECTS:}

Several methodologies for designing the repeater driven interconnect have been given in the literature. Broadly these methodologies can be classified as: delay centric and throughput (bits per second) centric. The primary objective of the delay-centric design is optimization of number and size of the repeaters to achieve minimum propagation delay. In the other method, these optimizations are carried out to achieve maximum possible throughput. Voltage-scaling is one of the most effective methods of containing power dissipation and propagation delay. Deodhar and Davis [15] considered voltage-scaled repeater system design. With the help of SPICE simulation results, they demonstrated that voltage scaling could control power dissipation in a repeater system. However, their approach being throughput-centric provides a limited picture of how voltage-scaling would affect a delay centric design. This paper deals primarily with the influence of voltage-scaling on the optimum number of repeaters in a delay-centric repeater-chain design for long interconnects.

The insertion of voltage-scaled repeaters in long interconnections have shown new and encouraging results in deep submicron technologies, it leads to a decrease in optimum number of repeaters required to be inserted in a long interconnect for delay minimization. Thus voltage scaled repeaters can enhance the performance of interconnects largely. The performance of the voltage scaled repeaters is analyzed using SPICE simulation tools. All interconnect parameters used in simulations are obtained from ITRS 2005 [16] as summarized in Table 1.

\section{COMPARATIVE ANALYSIS OF INTERCONNECTS}

In this section, the different parameters of an MWCNT interconnect is estimated and compared with the traditional $\mathrm{Cu}$ interconnect. All interconnect parameters used are obtained from ITRS 2005[18], as summarized in table I. In this paper, the diameter of MWCNT and $\mathrm{Cu}$ wire is set equal to minimum width of interconnects at each technology node.

Table 1. ITRS 2005 based simulation parameters

\begin{tabular}{|c|c|c|c|c|}
\hline \multicolumn{2}{|c|}{ Technology Node (nm) } & \multirow{2}{*}{$\begin{array}{l}32 \\
32\end{array}$} & \multirow{2}{*}{$\frac{22}{22}$} & \multirow{2}{*}{$\begin{array}{l}14 \\
14\end{array}$} \\
\hline & Width W (nm) & & & \\
\hline Local \& & $A / R$ & 2 & 2 & 2 \\
\hline \multirow[t]{5}{*}{ Intermediate } & ILD Thickness $\mathrm{t}_{\mathrm{ax}}(\mathrm{nm})$ & 54.4 & 39.6 & 25.2 \\
\hline & $\rho_{\mathrm{cu}}(\mu \Omega \mathrm{cm})$ & 4.83 & 6.01 & 8.19 \\
\hline & $\mathrm{C}_{\mathrm{Cu}}(\mathrm{pF} / \mathrm{m})$ & 144.93 & 131.01 & 111.83 \\
\hline & Width W (nm) & 48 & 32 & 21 \\
\hline & $\mathrm{A} / \mathrm{R}$ & 3 & 3 & 3 \\
\hline \multirow[t]{3}{*}{ Global } & ILD Thickness $t_{o x}(n m)$ & 110.4 & 76.8 & 52.5 \\
\hline & $\rho_{\mathrm{Cu}}(\mu \Omega \mathrm{cm})$ & 3.52 & 4.2 & 5.38 \\
\hline & $\mathrm{C}_{\mathrm{Cu}}(\mathrm{pF} / \mathrm{m})$ & 179.78 & 163.3 & 139.03 \\
\hline$\overline{\mathrm{K}_{\text {ILD }}}$ & & 2.25 & 2.05 & 1.75 \\
\hline
\end{tabular}

The aspect ratio (A/R) for global level interconnects in ITRS is in the range of 2.5-2.8. For convenience, we have used aspect ratio $(\mathrm{A} / \mathrm{R})=3$.

\section{RESULTS AND DISCUSSIONS:}

The values of R, L and C for Copper and MWCNT have been calculated through MATLAB. Propagation Delay in Copper and MWCNT interconnects for $32 \mathrm{~nm}, 22 \mathrm{~nm}$ technologies is calculated using SPICE simulation. Simulation is done here for different no. of repeaters „n“ viz. 1,3,5,7,9,11. Aspect ratio of the inverters used here in the buffer is 40 . Fig. 10 and 11 shows the comparison of $32 \mathrm{~nm}$ and $22 \mathrm{~nm}$ technologies nodes for delay respectively with various numbers of repeaters. From these results we observe that as we keep on increasing the number of repeaters in interconnect, the difference between the propagation delay for MWCNT and Copper keep on narrowing. The repeater Aspect Ratio has a fixed ratio of 40 . 


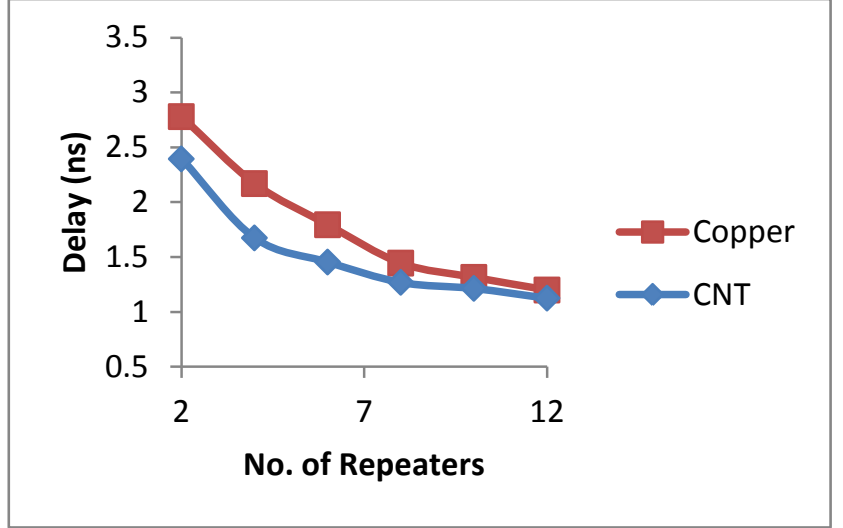

Fig.10. Comparison of delay between CNT and Copper for 32nm technology

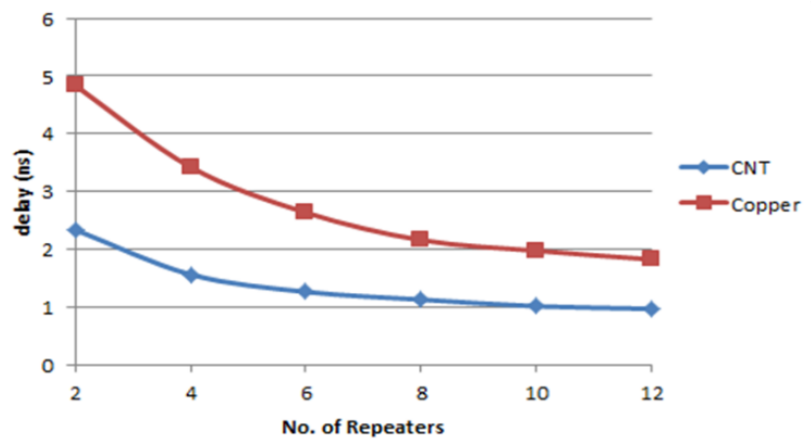

Fig.11. Comparison of delay between CNT and Copper for $22 \mathrm{~nm}$ technology

From fig. 12 and 13 gives the SPICE simulated results in terms of propagation delay for Copper and MWCNT Interconnects respectively for $32 \mathrm{~nm}$ and $22 \mathrm{~nm}$ technology nodes. It can be observed from these graphs that propagation delay reduces with voltage scaling for the same number of repeaters inserted in interconnects for both copper and MWCNT. This trend can be observed for both $32 \mathrm{~nm}$ and $22 \mathrm{~nm}$ technologies. Thus use of voltage scaling with optimum number of repeaters can enhance the performance of the VLSI circuits.

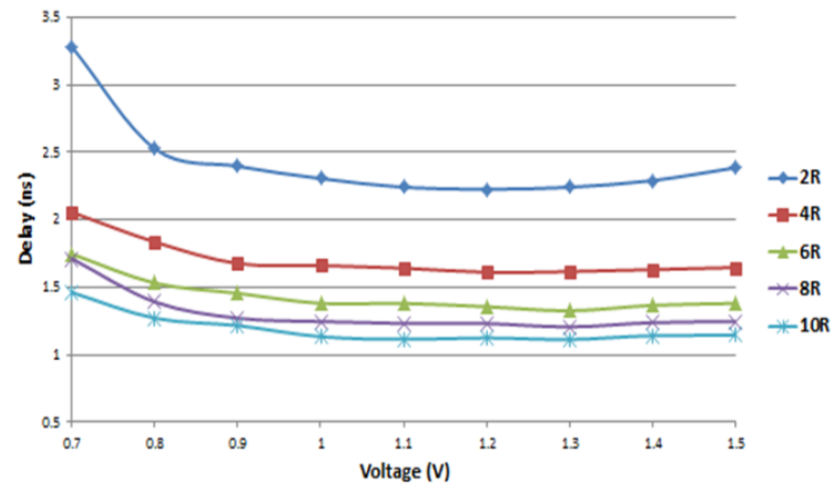

Fig.12 Variation of delay with voltage for Copper (32nm)

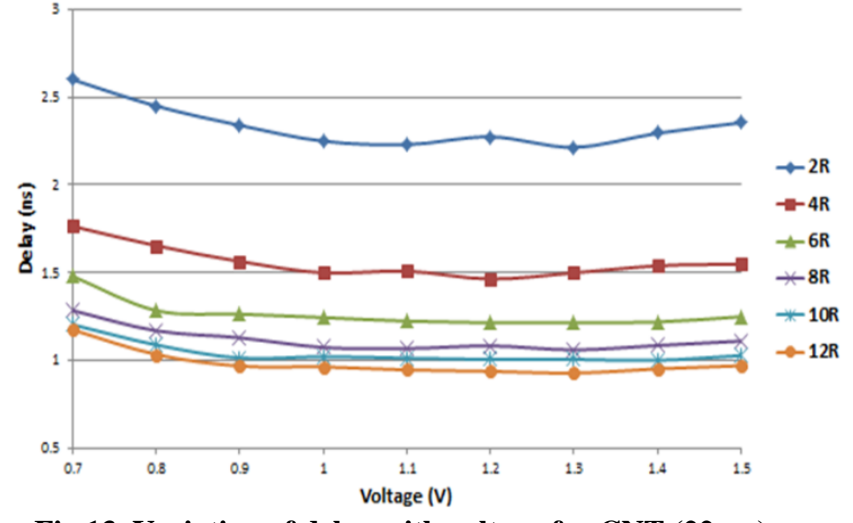

Fig.13. Variation of delay with voltage for CNT (22nm)

\section{CONCLUSION}

This paper shows the applicability of MWCNTs as an interconnect candidate in future design of integrated circuit. A comprehensive equivalent distributed circuit model of MWCNT and copper has been presented. The delay performance of MWCNT and copper interconnects have been compared for various number of repeaters. MWCNT interconnects shows significant improvement in performance as compared to copper interconnects due to low resistivity. Optimum delay can be achieved with the use of voltage scaled repeaters. It has been observed from results that propagation delay reduces with voltage scaling for same number of repeaters and lower delay can be achieved with less number of repeaters at high voltage as compared to more number of repeaters at less voltage.

\section{REFERENCES}

[1]. Mayank Kumar Rai, Sankar Sarkar, "Carbon Nano Tube as VLSI Interconnect”, Electronic Properties of Carbon Nanotubes, intech,2011

[2]. Steinhogl, W., Schindler, G., Steinlesberger, G., Traving, M., Engelhardt, M.: 'Comprehensive study of the resistivity of copper wires with lateral dimensions of $100 \mathrm{~nm}$ and smaller', J. Appl. Phys., 2005.

[3]. Naeemi et al. "Performance comparison between carbon nanotube and copper interconnects for giga scale integration (GSI)", Electron Device letters, vol. 26, No. 2, pp. 84-86, 2005.

[4]. H. Li, C. Xu, N. Srivastava and K. Banerjee, "Carbon Nanomaterials for Next-Generation Interconnects and Passives: Physics, Status and

Prospects," IEEE Trans. Electron Devices, vol. 56, no. 9, pp. 1799-1821, Sep. 2009.

[5]. K. Banerjee and N. Srivastava, "Are carbon nanotubes the future of VLSI interconnections?," in Proc. Des. Autom. Conf., 2006, pp. 809-814.

[6]. Rajeevan Chandel, S. Sarkar., Repeater insertion in global interconnects in VLSI circuits, Emerald Group Publishing Limited, Microelectronics International, 2005. 
[7]. N. Srivastava, H. Li, F. Kreupl, and K. Banerjee, “On the applicability of single-walled carbon nanotubes as VLSI interconnects," IEEE Trans. Nanotechnol., vol. 8, no. 4, pp. 542-559, Jul. 2009.

[8]. Tarun Parihar, Abhilasha Sharma, "A comparative study of Mixed CNT bundle with Copper for VLSI Interconnect at 32nm", International Journal of Engineering Trends and Technology (IJETT), April 2013.

[9]. H. J. Li, W. G. Lu, J. J. Li, X. D. Bai and C. Z. Gu, "Multichannel ballistic transport in multiwall carbon nanotubes," Phys. Rev. Lett., vol. 95, p. 86601, 19 August 2005.

[10]. H. Li, W. Y. Yin, K. Banerjee, and J. F. Mao, "Circuit modelling and performance analysis of multi-walled carbon nanotube interconnects," IEEE Trans. Electron Devices, vol. 55, no. 6, 2008.

[11]. A. Naeemi and J. D. Meindl, "Compact physical models for multiwall carbon-nanotube interconnects," IEEE Electron Device Lett., vol. 27, no. 5, pp. 338340, May 2006.

[12]. Y. Xu and A. Srivastava, "A Model of Multi-Walled Carbon Nanotube Interconnects," Proc 52nd IEEE Int. Midwest Sym. Cir. and Sys, 2009.

[13]. P. J. Burke, "Lüttinger Liquid Theory as a Model of the Gigahertz Electrical Properties of Carbon Nanotubes," IEEE Trans. Nanotechnology, vol. 1, no. 3, pp. 129-144, Sep. 2002.
[14]. V.V. Deodhar, et al., "Voltage-scaling and repeater insertion for highthroughput low-power interconnects", Proc. ISCAS'03 5, pp. v-349-352, 2003

[15]. Iman Madadi, Hossein Aghababa, and Behjat Forouzandeh, "Multi-Walled Carbon NanoTube Impedance”, IEEE International Conference, 2010.

[16]. P. G. Collins and P. Avouris, "Multishell conduction in multiwalled carbon nanotubes," Appl. Phys. A, Solids Surf., vol. 74, no. 3, pp. 29-332, Mar. 2002.

[17]. Ashok Srivastav, Yao Xu, Ashwani K. Sharma "Carbon nanotubes for next generation very large scale integration interconnects", journal of nanophotonics, vo. No. 4,041690, May 2010.

[18]. M. Gholipour, N. Masoumi: "Efficient inclusive analytical model for delay estimation of multi-walled carbon nanotube interconnects" IET Circuits Devices Syst., 2012.

[19]. Manoj Kumar Majumder, Nisarg D. Pandya, B. K. Kaushik, and S. K. Manhas "Analysis of MWCNT and Bundled SWCNT Interconnects: Impact on Crosstalk and Area", Electron Device Letters, IEEE, 2012

[20]. J. F. Xu, H. Li, W. Y. Yin, J. F. Mao, and L. W. Li, "Extraction of three-dimensional interconnects using element-by-element finite element method and preconditioned conjugate gradient technique," IEICE Trans. Electron., vol. E90C, no. 1, pp. 179-188, Jan. 2007. 